Randomized Controlled Trial

\title{
Immediate effects of diaphragmatic myofascial release on the physical and functional outcomes in sedentary women: A randomized placebo- controlled trial ${ }^{\text {is }}$
}

\author{
Débora Fortes Marizeiro, Ana Carolina Lins Florêncio, Ana Carla Lima Nunes, \\ Nataly Gurgel Campos, Pedro Olavo de Paula Lima* \\ Department of Physical Therapy, Fortaleza, Ceará, CE, Brazil
}

\section{A R T I C L E I N F O}

\section{Article history:}

Received 3 June 2017

Received in revised form

10 October 2017

Accepted 15 October 2017

\section{Keywords:}

Diaphragm

Myofascial release

Physical therapy

\begin{abstract}
A B S T R A C T
Background: Although diaphragmatic myofascial release techniques are widely used in clinical practice, few studies have evaluated the simultaneous acute effects of these techniques on the respiratory and musculoskeletal systems.

Objective: To evaluate the immediate effects of diaphragmatic myofascial release in sedentary women on the posterior chain muscle flexibility; lumbar spine range of motion; respiratory muscle strength; and chest wall mobility.

Design: A randomized placebo-controlled trial with concealed allocation, intention-to-treat analysis, and blinding of assessors and participants.

Participants: Seventy-five sedentary women aged between 18 and 35 years.

Intervention: The sample was randomly allocated into one of two groups; the experimental group received two diaphragmatic myofascial release techniques in a single session, and the control group received two placebo techniques following the same regimen.

Outcomes measures: The primary outcome was chest wall mobility, which was analyzed using cirtometry. The secondary outcomes were flexibility, lumbar spine range of motion, and respiratory muscle strength. Outcomes were measured before and immediately after treatment.

Results: The manual diaphragm release techniques significantly improved chest wall mobility immediately after intervention, with a between-group difference of $0.61 \mathrm{~cm}(95 \% \mathrm{Cl}, 0.12-1.1)$ for the axillary region, $0.49 \mathrm{~cm}(95 \% \mathrm{CI}, 0.03-0.94)$ for the xiphoid region, and $1.44(95 \% \mathrm{CI}, 0.88-2.00)$ for the basal region. The techniques also significantly improved the posterior chain muscle flexibility, with a betweengroup difference of $5.80 \mathrm{~cm}(95 \% \mathrm{CI}, 1.69-9.90)$. All movements except flexion of the lumbar spine significantly increased. The effects on respiratory muscle strength were non-significant.

Conclusion: The diaphragmatic myofascial release techniques improve chest wall mobility, posterior chain muscle flexibility, and some movements of the lumbar spine in sedentary women. These techniques could be considered in the management of people with reduced chest wall and lumbar mobility. Trial registration: NCT03065283.
\end{abstract}

๑) 2017 Elsevier Ltd. All rights reserved.

\footnotetext{
t The study was recorded in a clinical trials registry (clinicaltrials.gov NCT03065283)

* Corresponding author. Federal University of Ceara, Department of Physical Therapy, Alexandre Baraúna Street 949, 60430-160, Fortaleza, CE, Brazil. Tel.: +55 8533668632.

E-mail addresses: debora.fortes@hotmail.com (D.F. Marizeiro), carollinsf@gmail com (A.C.L. Florêncio), aclnunes@gmail.com (A.C.L. Nunes), natgurgel@yahoo.com. br (N.G. Campos), pedrolima@ufc.br (P.O.P. Lima).
}

\section{Introduction}

The diaphragm is classically described as a thin, flat muscle that separates the thoracic and abdominal cavities. The lumbar (or vertebral) portion of the diaphragm muscle is the most posterior, robust, and strong, with vertical fibers and bilateral arrangement at the side of the lumbar spine (Williams et al., 1989). During inspiration, pressure increases in the abdomen and decreases in the thorax (Barr et al., 2005). Intra-abdominal pressure is determined 
by thoracic and abdominal mechanics and respiratory muscle strength (Hodges et al., 2005). The diaphragm is also involved in motor control of the spine; it plays a key role in spine stability during postural control in movements with large amplitude, such as diaphragmatic contraction, during which there is a subsequent increase in the abdominal pressure that stimulates an activation of the pelvic floor muscles followed by eccentric contraction of the abdominal wall muscles (Nason et al., 2012; Kolar et al., 2010).

Sedentary behavior is a risk factor for several diseases, including musculoskeletal and respiratory disorders (Overgaard et al., 2013; Chen et al., 2009). The most striking feature of this type of respiratory disorder is the structural change of the muscular parenchyma. In the diaphragm, the muscle fibers that are usually vertically arranged in the apposition zone may become more transversally oriented (Sollanek et al., 2015). This makes the diaphragm's contraction less effective, decreasing its pressuregenerating capacity (Lieber and Ward, 2011; Wakeling et al., 2006). As a consequence, diaphragmatic mobility is reduced, which is a major risk factor for pulmonary diseases (Yamaguti et al., 2009). In addition, musculoskeletal changes inherent to the sedentary lifestyle contribute to increased chest wall stiffness, hindered rib cage expansion, increased breathing work, and reduced respiratory function (Hochhegger et al., 2012).

Given the interdependent relationship between the respiratory and musculoskeletal systems, various manual techniques have been proposed for the management of people with reduced chest wall and lumbar mobility. A common goal is increasing the mobility of the thoracic structures involved in respiratory mechanics (Chaitow et al., 2002; Somers, 2005). Manual myofascial release is an intervention intended to indirectly stretch the diaphragmatic muscle fibers to reduce tension generated by trigger points, normalize fiber length, and promote greater muscle contraction effectiveness. The techniques are generally applied with low-speed movements, and the action mechanism is based on the sensibility of Golgi tendon organs (Teles et al., 2013). Diaphragmatic release techniques are widely known and used in clinical practice, and no contraindications or side effects have been reported in the literature (González-Álvarez et al., 2016; Rocha et al., 2015).

However, few studies have evaluated the simultaneous acute effects of these techniques on the respiratory and musculoskeletal systems. The aim of this study was to evaluate the immediate effects of diaphragmatic myofascial release in healthy sedentary women on chest wall mobility, posterior chain muscle flexibility, lumbar spine range of motion (ROM), and respiratory muscle strength.

\section{Methods}

\subsection{Design}

A randomized placebo-controlled trial was conducted in the Department of Physical Therapy of the Federal University of Ceará from 2015 to 2016. This study was approved by the Research Ethics Committee of the Federal University of Ceará (\#047/2011), and all participants signed a written consent form. The sample was randomly allocated into two groups via drawing from an opaque envelope by one researcher who was not involved in the study. Both groups received interventions in a single session, following the same regimen. The assessors responsible for outcome measurement and data analysis were blinded as to group assignment.

\subsection{Participants}

Participants were recruited from a list of students at the Federal University of Ceará and included only subjects who were female, sedentary (self-reported), and aged 18-35. We selected only women because the ventilatory mechanics between genders are physiologically different, perhaps due to men's larger airway caliber, greater chest wall dimension, force of contraction of respiratory muscles, and even differences in breathing patterns. Exclusion conditions were: a) cardiovascular disease; b) obstructive or restrictive respiratory disease; c) smokers and ex-smokers; d) thoracic scoliosis; e) participation or past participation in any choir group; and f) treatment with speech therapy in the last 12 months.

\subsection{Outcome measures}

The primary outcome was chest wall mobility, and the secondary outcomes were posterior chain muscle flexibility, lumbar spine ROM, and respiratory muscle strength. Outcomes were measured in both groups before and immediately after the single session. Chest wall mobility was evaluated with cirtometry using a simple tape measure, marked in centimeters, around the thorax and abdomen. Maximum inspiration and expiration were observed to obtain the thoracic-abdominal amplitude coefficient, which is characterized by the difference between these values in the axillary (CA-Ax), xiphoid (CA-Xif), and basal (CA-Ba) regions (Pedrini et al., 2013). The sit-and-reach test with Wells bank was used to evaluate the posterior chain muscle flexibility (i.e., erector spinae, gluteus maximus, ischiotibials, triceps surae, and foot intrinsic muscles), with three replicates, recording the largest distance achieved (Bertolla et al., 2007). Lumbar spine ROM was assessed with a goniometer. Flexion, extension, and right/left side-flexion were measured. Respiratory muscle strength was evaluated with the $\mathrm{MR}^{\circledR}$ brand analogue manovacuometer in the orthostatic position. The examiner verbally requested maximum inspiration and expiration to measure maximal inspiratory pressure (MIP) and maximal expiratory pressure (MEP). Each measurement was carried out three times, recording the best result of the three (ATS, 2002).

\subsection{Interventions}

Participants in both groups received a simple session of experimental or placebo treatment. The same physiotherapist performed the intervention in both groups, to ensure similar application of the experimental and placebo interventions.

The control group received a sham protocol. The manual contacts, duration, and positioning of the physiotherapist and participant were the same as in the experimental group, but the physiotherapist maintained only a light touch on the anatomical landmarks, without exerting pressure or traction. This was intended to blind all participants to their group assignment during the study.

The experimental group (EG) received two diaphragmatic myofascial release techniques. The first intervention technique performed was the "lift diaphragm," to stretch the peripheral fibers of the diaphragm muscle. Participants lay supine with relaxed limbs, and the physiotherapist was positioned at the subject's head. The physiotherapist made manual contact with the lower edge of the costal arch. In the inspiratory phase, the physiotherapist gently pulled the points of contact with both hands slightly laterally and in the direction of the head, accompanying the elevation of the ribs. During exhalation, the physiotherapist deepened contact toward the inner costal margin, maintaining resistance. The technique was performed in two sets of 10 deep breaths on each side, with a 1-min interval between them (Quef, 2008).

The second technique was relaxation of the diaphragm pillars, which aims to promote the rhythmic stretching of the psoas and diaphragm insertions. Patients lay prone with the physiotherapist standing beside them, placing the cephalic hand on the last ribs and 
the caudal hand on the popliteal fossa. The physiotherapist applied a divergent force by pulling away the hands, without slide, during the exhalation phase and decreased the force in the inspiration phase. This maneuver was performed for $1 \mathrm{~min}$ on each side (Quef, 2008).

The control group received two placebo techniques with the same position of physiotherapist and participant, verbal commands, number of repetitions, and manual contact as the intervention group. However, in the two placebo techniques, only manual contacts were performed, with a light touch on the skin, using the same anatomical points as the intervention group but without application of force (Licciardone and Russo, 2006).

\subsection{Statistical analysis and sample size}

The sample size was calculated using Research Randomizer online, which accepts the anticipated data from each group to determine sample size. A total of 38 participants was recommended for a parallel study with two groups in order to obtain a difference of treatment of 2.48 (SD 2.41) cm in axillary cirtometry with a power of $80 \%$ and $\alpha$ of $5 \%$ (González-Álvarez et al., 2016). This study predicted a $20 \%$ sample loss for the calculation. Data were analyzed in the SPSS version 20.0 program with a significance level of $\alpha \leq 0.05$. Data were normally distributed according to the Kolmogorov-Smirnov test. A paired $t$-test was used for intragroup analysis, and an independent Student's t-test was used for intergroup analysis. Data are represented in mean between-group difference with a $95 \% \mathrm{CI}$, and analysis was by intention to treat.

\section{Results}

Fig. 1 shows the flow of participants through the study. One hundred and ten women were invited to participate, but 35 were subsequently excluded. The analyzed sample consisted of 75 participants who were randomly allocated into the EG $(n=50)$ and CG $(\mathrm{n}=25)$. The baseline characteristics of the participants are presented in Table 1, which shows that the groups are balanced. All participants received all scheduled treatments as allocated by the randomization process and were analyzed in those groups (i.e., intention-to-treat analysis).

\subsection{Chest wall mobility}

Table 2 presents the chest wall mobility data of both groups. The average immediate effect after the single session was a betweengroup difference in favor of the EG of $0.61 \mathrm{~cm}(95 \% \mathrm{CI}, 0.12-1.1)$ for the axillary region, $0.49 \mathrm{~cm}(95 \% \mathrm{CI}, 0.03-0.94)$ for the xiphoid region, and $1.44 \mathrm{~cm}(95 \% \mathrm{CI}, 0.88-2.00)$ for the basal region.

\subsection{Respiratory muscle strength}

Table 2 presents the respiratory muscle strength data of both groups. Despite intragroup analysis in favor of the EG, there was no significant difference between the groups after the intervention, with a between-group difference of $5.00 \mathrm{~cm} / \mathrm{H}_{2} \mathrm{O}(95 \% \mathrm{CI}$, -6.47-16.47) for MIP and $0.70 \mathrm{~cm} / \mathrm{H}_{2} \mathrm{O}(95 \% \mathrm{CI},-10.34-11.74)$ for MEP. The immediate effects on respiratory muscle strength were non-significant.

\subsection{Flexibility and lumbar spine range of motion (ROM)}

The posterior chain muscle flexibility data of both groups are presented in Table 3. The manual diaphragm release techniques also significantly improved the flexibility, with a between-group difference of $5.80 \mathrm{~cm}(95 \% \mathrm{CI}, 1.69-9.90)$ in favor of the EG. The lumbar spine ROM data of both groups are presented in Table 3. All movements except flexion of the lumbar spine significantly increased.

\section{Discussion}

In our study, the diaphragmatic lift technique and the relaxation maneuver of the diaphragm pillars significantly increased chest wall mobility, lumbar spine ROM, and posterior chain muscle flexibility immediately after intervention. However, no significant change was observed in respiratory muscle strength and lumbar flexion ROM.

\subsection{Chest wall mobility}

We observed a significant increase in chest wall mobility for three of the four measurements (excluding flexion) in the EG immediately after the techniques. This may have occurred due to decreasing stiffness of the diaphragm muscle, which allowed a greater rib cage motion and improved the length-tension relationship (Zaslavsky and Gus, 2002). Some studies (Noll et al., 2008, 2009) have used diaphragm stretching techniques to increase the chest wall mobility because these can improve lung expandability.

In the study by González-Álvarez et al. (2016), a technique similar to the diaphragm lift was applied, but over a longer time (about 5-7 $\mathrm{min}$ ) and with a disconnected ultrasound in the placebo group. They observed a significant difference in chest wall mobility only at the xiphoid level; this differs from our study, which found differences at all levels. This divergence may have occurred due to differing patient positions during application of the techniques. The authors adopted a sitting position that does not favor the elongation of the muscular fibers of the diaphragm, and they excluded sedentary individuals what might also contribute to a difference in results with our study. The results of another study (Braga et al., 2016) are in agreement with our results related to chest wall mobility immediately after two diaphragmatic release techniques in sedentary young women. This may be because the techniques performed in both studies used the same protocol in a similar population and the outcomes were measured by the same evaluation tool (cirtometry).

The effects of the Global Postural Reeducation method on respiratory muscle strength and thoracoabdominal mobility were evaluated in 20 individuals, and there was a significant increase at three levels of chest wall mobility in the EG. The intervention protocol used by the Global Postural Reeducation method consisted of a program of stretching exercises of the respiratory muscles, including the diaphragm. Therefore, the effects of this method were similar to those of the manual techniques used in our study, but the authors evaluated the outcomes over the medium term (Moreno et al., 2007).

\subsection{Respiratory muscle strength}

There was no a significant difference in MIP and MEP values between the groups. In the literature, diaphragm release techniques are indicated to improve the length-tension relationship of the muscle fibers to increase respiratory performance. The respiratory muscle strength should be reflected in the maximum pressure values generated because the myofascial restriction reduction of the diaphragm muscle could improve respiratory biomechanics, consequently increasing lung expandability and pressure gradients, which was not evidenced in our study (González-Álvarez et al., 2016). The characteristics of the sample can explain these results; these were sedentary women that were nevertheless healthy in relation to the pulmonary condition. Therefore no large changes in 


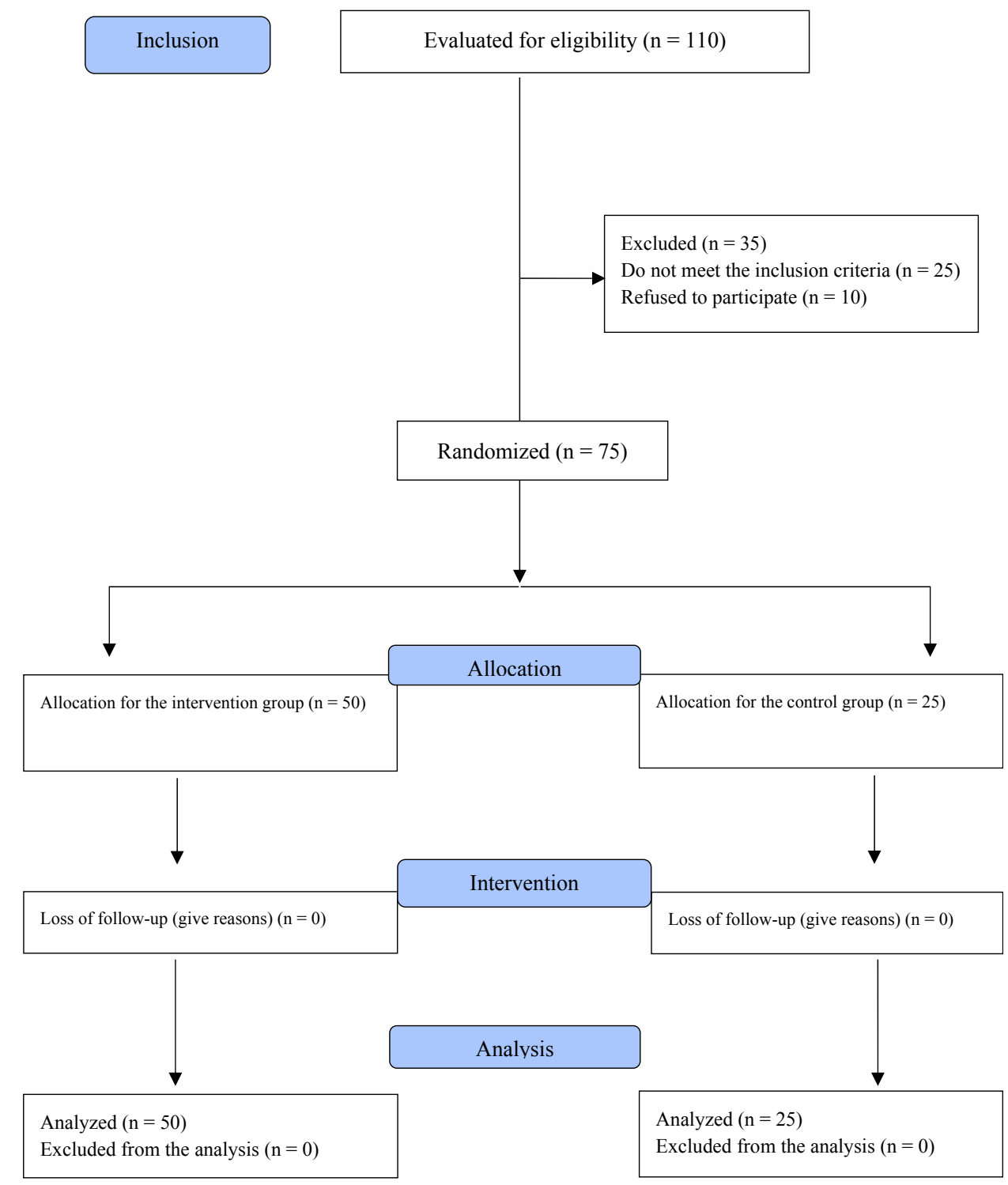

Figure 1. Flowchart of the selection, allocation and intervention of the sample according to CONSORT (Consolidated Standards of Reporting Trials).

Fig. 1. Flowchart of the selection, allocation and intervention of the sample according to CONSORT (Consolidated Standards of Reporting Trials).

Table 1

Characteristics of the sample in both groups.

\begin{tabular}{llll}
\hline Variables & Intervention Group & Control Group & $p$ \\
\hline Age (years) & $21.24 \pm 2.86$ & $21.12 \pm 2.12$ & 0.839 \\
Height $(\mathrm{m})$ & $1.62 \pm 0.06$ & $1.70 \pm 1.61$ & 0.599 \\
Weight $(\mathrm{kg})$ & $60.14 \pm 8.95$ & $62.80 \pm 12.38$ & 0.345 \\
$\mathrm{BMI}^{£}\left(\mathrm{Kg} / \mathrm{m}^{2}\right)$ & $23.12 \pm 3.04$ & $23.97 \pm 4.26$ & 0.382 \\
\hline
\end{tabular}

${ }^{*} \mathrm{BMI}=$ Body Mass Index.

pulmonary pressures were expected; such changes may hypothetically happen when the technique is applied for people with respiratory diseases (Paulin et al., 2003). Analyzing only the immediate effects does not allow for capturing changes in muscular strength, since this physical capacity is modified over time.
Rocha et al. (2015) analyzed the effects of the manual diaphragm release technique on the diaphragmatic mobility, exercise capacity, respiratory muscle strength, and abdominal and chest wall kinematics in 20 patients with chronic obstructive pulmonary disease (COPD). They observed significant improvements in diaphragmatic mobility, exercise capacity, and inspiratory capacity but no benefit in respiratory muscle strength. Some methodological differences may explain these findings. First, the sample contained people with respiratory disease, and the technique was performed six times on nonconsecutive days, but the acute effects were also assessed. Furthermore, outcome measures were evaluated with different assessment tools, such as ultrasonography; the 6-min walk test; optoelectronic plethysmography; and the digital manometer. Similar results to our study were evidenced, as that experimental group obtained an increase in pulmonary pressures after the 
Table 2

Intra and intergroup differences in the chest wall mobility and inspiratory/expiratory muscle strength.

\begin{tabular}{|c|c|c|c|c|c|}
\hline Variables & $\begin{array}{l}\text { Intragroup EG } \\
(\mathrm{n}=50)\end{array}$ & $p$ & $\begin{array}{l}\text { IntragroupCG } \\
(\mathrm{n}=25)\end{array}$ & $p$ & Mean of intergroup differences \\
\hline \multicolumn{6}{|c|}{ Caaxillary (cm) } \\
\hline Pre- & $2.87 \pm 0.93$ & $0.001^{*}$ & $2.78 \pm 1.08$ & 0.161 & $0.09[-0.38$ to 0.57$]$ \\
\hline Post- & $3.47 \pm 1.00$ & & $2,86 \pm 0,99$ & & $0.61[0.12$ to 1.1$]$ \\
\hline \multicolumn{6}{|c|}{ Caxiphoid (cm) } \\
\hline Pre- & $2.82 \pm 0,68$ & $0.001^{*}$ & $3.00 \pm 0.98$ & 0.627 & $-0.18[-0.57$ to 0.20$]$ \\
\hline & $3.45 \pm 0,97$ & & $2.96 \pm 0.85$ & & $0.49[0.03$ to 0.94$]$ \\
\hline \multicolumn{6}{|c|}{ Cabasal (cm) } \\
\hline Pre- & $2.85 \pm 1,01$ & $0.001^{*}$ & $2.28 \pm 0.87$ & 0.664 & $0.57[-0.09$ to 1.04$]$ \\
\hline Post- & $3.70 \pm 1.29$ & & $2.26 \pm 0.76$ & & $1.44[0.88$ to 2.00$]$ \\
\hline \multicolumn{6}{|c|}{$\operatorname{MIP}\left(\mathbf{c m} / \mathbf{H}_{2} \mathbf{O}\right)$} \\
\hline Pre- & $96.70 \pm 26.25$ & $0.001^{*}$ & $102.80 \pm 28.21$ & 0.714 & $-6.10[-19.23$ to 7.03$]$ \\
\hline Post- & $107.40 \pm 20.23$ & & $102.400 \pm 29.05$ & & $5.00[-6.47$ to 16.47$]$ \\
\hline \multicolumn{6}{|c|}{$\operatorname{MEP}\left(\mathrm{cm} / \mathrm{H}_{2} \mathbf{O}\right)$} \\
\hline Pre- & $76.90 \pm 18.5$ & $0.001^{*}$ & $89.29 \pm 27.67$ & 0.425 & $-12.30[-23.01$ to -1.58$]$ \\
\hline Post- & $90.70 \pm 19.79$ & & $90.00 \pm 27.53$ & & $0.70[-10.34$ to 11.74$]$ \\
\hline
\end{tabular}

* Significant at the level of $\mathrm{p} \leq 0.05$. EG: experimental group. CG: control group. Ca: Cirtometry.

Table 3

Intra and intergroup differences in the posterior flexibility and lumbar spine range of motion.

\begin{tabular}{|c|c|c|c|c|c|}
\hline Variables & Intragroup EG $(\mathrm{n}=50)$ & $p$ & $\begin{array}{l}\text { Intragroup CG } \\
(\mathrm{n}=25)\end{array}$ & $p$ & Mean of intergroup differences \\
\hline \multicolumn{6}{|c|}{ Flexibility (cm) } \\
\hline Pre- & $29.95 \pm 9.53$ & $0.001^{*}$ & $23.74 \pm 7.58$ & $0.007^{*}$ & $3.21[-1.15$ to 7.57$]$ \\
\hline Post- & $30.08 \pm 8.68$ & & $24.28 \pm 7.84$ & & $5.80[1.69$ to 9.90$]$ \\
\hline \multicolumn{6}{|c|}{ Flexion $\left({ }^{\circ}\right)$} \\
\hline Pre- & $98.30 \pm 13.87$ & $0.001^{*}$ & $102.60 \pm 13.00$ & 0.114 & $-4.30[-10.93$ to 2.33$]$ \\
\hline \multicolumn{6}{|c|}{ Extension $\left({ }^{\circ}\right)$} \\
\hline Pre- & $28.50 \pm 7.53$ & $0.001^{*}$ & $35.28 \pm 9.78$ & 0.185 & $-6.78[-5.69$ to 188$]$ \\
\hline Post- & $35.28 \pm 3.78$ & & $30.52 \pm 8.18$ & & $4.76[0.22$ to 9.29$]$ \\
\hline \multicolumn{6}{|c|}{ Right side-flexion $\left({ }^{\circ}\right)$} \\
\hline Pre- & $30.58 \pm 7.09$ & $0.001^{*}$ & $31.48 \pm 7.48$ & 0.327 & $-0,90[-4,42$ to 2,62$]$ \\
\hline \multicolumn{6}{|c|}{ Left side-flexion $\left(^{\circ}\right)$} \\
\hline Pre- & $30.88 \pm 6.23$ & $0.001^{*}$ & $33.00 \pm 8.03$ & 0.792 & $-2.12[-5.47$ to 1.23$]$ \\
\hline Post- & $37.18 \pm 6.97$ & & $33.08 \pm 7.66$ & & $4.10[0.57$ to 7.62$]$ \\
\hline
\end{tabular}

${ }^{*}$ Significant at the level of $\mathrm{p} \leq 0.05$. EG: experimental group. CG: control group.

techniques; however, this increase was not statistically significant compared to the control group.

Novaes et al. (2013) also evaluated the effects of a diaphragmatic release technique applied only once over 3 min in sedentary men and women, and no statistical difference was found for MIP and MEP values. Another clinical trial was conducted with 43 participants of both genders divided into four groups (control; manipulation; mobilization; and manipulation with mobilization), with spinal manipulation of the third cervical vertebra (C3) and mobilization of the soft tissues of the diaphragm. No significant difference was observed in MIP and MEP values immediately after intervention, which is in line with our results (Carvalho et al., 2011).

\subsection{Flexibility}

There was a significant increase in posterior chain muscle flexibility in favor of the EG immediately after the techniques. This may be because the diaphragm pillars technique was performed with one of the physiotherapist's hands at the popliteal region where the hamstring tendon is inserted, and the sit-and-reach test is more sensitive to changes in the hamstring length than to changes in the lumbar muscles (Melo et al., 2011).

All of results corroborate the majority of studies published about the topic, most likely because these studies applied similar techniques. Valenza et al. (2015) recruited 60 young adults with short- hamstring syndrome and applied a diaphragm stretching technique similar to the diaphragm lift but with different positioning and duration. A placebo technique was performed in the control group with a disconnected ultrasound in the xiphoid process for $5 \mathrm{~min}$. Authors observed a between-groups mean difference of $-5.07 \mathrm{~cm}(95 \% \mathrm{CI},-7.41-2.19)$ in the forward flexion distance test and $-7.41^{\circ}(95 \% \mathrm{CI},-8.45-3.81)$ in the popliteal angle test.

In another study involving 80 healthy individuals, the diaphragm lift technique was compared to a placebo ultrasound, and a significant increase in posterior chain muscle flexibility was observed (González-Álvarez et al., 2016). In addition, certain authors argued that interventions in the diaphragm may have repercussions on distant structures due to the myofascial tension transmission of the muscle fibers inserted into the inner face of the xiphoid process and lumbar vertebrae (Hamaoui et al., 2007; Dias and Gómez-Conesa, 2008).

\subsection{Lumbar spine ROM}

Our study found that movements of the lumbar spine significantly increased, except that despite the improvement in flexibility, lumbar flexion did not increase. Other studies have noted that all lumbar movements increase immediately after soft tissue mobilization techniques; however, the lumbar flexion ROM has been evaluated with the Schober test, a specific measure usually 
performed in patients with ankylosing spondylitis (GonzálezÁlvarez et al., 2016; Valenza et al., 2015).

\subsection{Clinical implications and study limitations}

Many sedentary people experience impairment of the chest wall mobility, posterior chain muscle flexibility, respiratory muscle strength, and lumbar spine ROM compared to physically active people. The diaphragmatic myofascial release techniques apply manual pressure under the costal margin, to stretch the rib cage and the insertional fibers of the diaphragm. Only one session of these techniques led to immediate improvements in chest wall mobility, flexibility, and lumbar ROM. We suggest future studies to test the effects of these techniques in populations with other health conditions. A limitation of this study was the impossibility of blinding the physiotherapist who administered the interventions. The outcome measures were only evaluated immediately after intervention, which may also be considered a limitation.

\section{Conclusion}

The present study has demonstrated that the diaphragmatic release techniques improve chest wall mobility, posterior chain muscle flexibility, and lumbar spine ROM in sedentary young women. No change was observed in respiratory muscle strength and lumbar flexion. These techniques can be used as an effective therapeutic tool with an immediate response, suggesting that they should be considered in the management of people with impairment of chest wall and lumbar mobility. The results of this study provide new data to assist therapeutic approaches.

\section{Funding}

This research did not receive any specific grant from funding agencies in the public, commercial, or not-for-profit sectors.

\section{Conflicts of interest}

None.

\section{References}

American Thoracic Society/European Respiratory Society ATS/ERS, 2002. Statement on respiratory muscle testing. Am. J. Respir. Crit. Care Med. 166, 518-624.

Barr, K.P., Griggs, M., Cadby, T., 2005. Lumbar stabilization: core concepts and current literature, part 1. Am. J. Phys. Med. Rehabil. 84, 473-480.

Bertolla, F.I., Baroni, B.M., Junior, E.C.P.L., Oltramari, J.D., 2007. Effects of a training program using the Pilates method in flexibility of sub-20 indoor soccer athletes. Rev. Bras. Med. Esporte 13, 222-226.

Braga, D.K.A.P., Marizeiro, D.F., Florêncio, A.C.L., Teles, M.D., Silva, I.C., Júnior, F.F.U.S., et al., 2016. Manual therapy in diaphragm muscle: effect on respiratory muscle strength and chest mobility. Man. Ther. Posturology Rehabil. J. 14, 302-308.

Carvalho, A.R., Silva, S.L., Oliveira, A.P., 2011. Efeito imediato de duas técnicas de terapia manual sobre a pressão inspiratória máxima em indivíduos saudáveis: ensaio clínico. Ter. Man. 9, 143-149.

Chaitow, L., Bradley, D., Gilbert, C., 2002. Multidisciplinary Approaches to Breathing Patterndisorders, first ed. Churchill Livingstone, London.

Chen, S.M., Liu, M.F., Cook, J., Bass, S., Lo, S.K., 2009. Sedentary lifestyle as a risk factor for low back pain: a systematic review. Int. Arch. Occup. Environ. Health 82, 797-806. https://doi.org/10.1007/s00420-009-0410-0.

Dias, R.S., Gómez-Conesa, 2008. Síndrome de los isquiotibiales acortados. Fisioterapia 30, 186-193.
González-Álvarez, F.J., Valenza, M.C., Torres-Sánchez, I., Cabrera-Martos, I., Rodríguez-Torres J,Castellote-Caballero, Y., 2016. Effects of diaphragm stretching on posterior chain muscle kinematics and rib cage and abdominal excursion: a randomized controlled trial. Braz J. Phys. Ther. 20, 405-411.

Hamaoui, A., Bozec, S.L., Poupard, L., Bouisset, S., 2007. Does postural chain muscular stiffness reduce postural steadiness in a sitting posture? Gait Posture $25,199-204$.

Hochhegger, B., De Meireles, G.P., Irion, K., Zanetti, G., Garcia, E., Moreira, J., 2012 The chestand aging: radiological findings. J. Bras. Pneumol. 38, 656-665.

Hodges, P.W., Eriksson, A.E., Shirley, D., Gandevi, S.C., 2005. Intra-abdominal pressure increases stiffness of the lumbar spine. J. Biomech. 38, 1873-1880.

Kolar, P., Sulc, J., Kyncl, M., 2010. Stabilizing function of the diaphragm: dynamic MRI and synchronized spirometric assessment. J. Appl. Physiol. 109, 1064-1071.

Licciardone, J.C., Russo, D.P., 2006. Blinding protocols, treatment credibility, and expectancy: methodologic issues in clinical trials of osteopathic manipulative treatment. J. Am. Osteopath Assoc. 106, 457-463.

Lieber, R.L., Ward, S.R., 2011. Skeletal muscle design to meet functional demands. Philos. Trans. R. Soc. Lond B Biol. Sci. 366 (1570), 1466-1476.

Melo, L.M.O., Martins-Costa, H.C., Araújo, S.R.S., Menzel, H.J., Chagas, M.H., 2011 Validade de testes utilizados para medir a flexibilidade dos músculos posteriores da coxaem estudantes universitários. Bras Mov 19, 52-60.

Moreno, M.A., Catai, A.M., Teodori, R.M., Borge, B.L.A., Cesar, M.C., Silva, E., 2007. Effect of a muscle stretching program using the Global Postural Re-education method on respiratory muscle strength and thoracoabdominal mobility of sedentary young males. J. Bras. Pneumol. 33, 679-686.

Nason, L.K., Walker, C.M., McNeeley, M.F., Burivong, W., Fligner, C.L., Godwin, J.D., 2012. Imaging of the diaphragm: anatomy and function. Radiographics 32, 51-70. https://doi.org/10.1148/rg.322115127.

Noll, D.R., Degenhardt, B.F., Johnson, J.C., Buró, S.A., 2008. Immediate effects of osteopathic manipulative treatment in elderly patients with chronic obstructive pulmonary disease. J. Am. Optom. Assoc. 108, 251-259.

Noll, D.R., Degenhardt, B.F., Johnson, J.C., Burt, S.A., 2009. The immediate effect of individual manipulation techniques on pulmonary function measures in persons with COPD. Osteopath Med. Prim. Care 3, 9.

Novaes, P.A., Sanchez, E.G.M., Sanchez, H.M., 2013. Medida das pressões respiratórias máximas em jovens saudáveis antes e após manobra de liberação diafragmática. Rev Inspirar Mov Saúde 5 (2), 1-5.

Overgaard, K., GjerndrupAagaard, P., Grøntved, A., Nielsen, K., Dahl-Petersen, I.K. Aadahl, M., 2013. Sedentary behaviour in Denmark is growing and is a possible independent risk factor. Ugeskr. Laeger 175, 2631-2635.

Paulin, E., Brunetto, A.F., Carvalho, C.R.F., 2003. Effects of a physical exercise program designed to increase thoracic expansion in chronic obstructive pulmonary disease patients. J. Pneumol. 29, 287-294.

Pedrini, A., Gonçalves, M.A., Leal, B.E., Yamaguti, W.P.S., Paulin, E., 2013. Comparison between the measures of thoracoabdominal cirtometry in supine and standing. Fisioter. Pesq. 20, 373-378.

Quef, B.H., 2008. Técnicas Osteopáticas Viscerais. Livraria Santos Editora, São Paulo. Rocha, T. Souza, H., Brandão, D.C., Rattes, C., Ribeiro, L., Campos, S.L., 2015. The Manual Diaphragm Release Technique improves diaphragmatic mobility, inspiratory capacity and exercise capacity in people with chronic obstructive pulmonary disease: arandomised trial. J. Physiother. 61, 182-189.

Sollanek, K.J., Smuder, A.J., Wiggs, M.P., Morton, A.B., Koch, L.G., Britton, S.L., Powers, S.K., 2015. Role of intrinsic aerobic capacity and ventilator-induced diaphragm dysfunction. J. Appl. Physiol. 118, 849-857. https://doi.org/10.1152/ japplphysiol.00797.2014.

Somers, D., 2005. An Osteopathic Approach to Diagnosis and Treatment, third ed. Lippincott Williams\& Wilkins, Philadelphia.

Teles JAA, Oliveira RPM, Loiola PRR 2013 Liberação diafragmática para ganho em flexão de cadeia muscular posterior. Fisiobrasil. Acessed in: 28 de Dezembro de 2016 http://fisiobrasil.com.br/main.asp?link=amateria\&id=4231.

Valenza, M.C., Cabrera-Martos, I., Torres-Sánchez, I., Garcés-García, A., MateosToset, S., Valenza-Demet, G., 2015. The immediate effects of doming of the diaphragm technique in subjects with short hamstring syndrome. A Randomized Control. Trial Rehabil. 24, 342-348.

Wakeling, J.M., Uehli, K., Rozitis, A.I., 2006. Muscle fibre recruitment can respond to the mechanics of the muscle contraction. J. R. Soc. Interface 3 (9), 533-544.

Williams, P.L., Warwick, R., Dyson, M., Bannister, L.H., 1989. Gray's Anatomy, 37th edition. Churchill Livingstone, London.

Yamaguti, W.S., Paulin, E., Salge, J.M., Chammas, M.C., Cukier, A., Carvalho, C.R.F.D. 2009. Diaphragmatic dysfunction and mortality in patients with COPD. J. Bras. Pneumol. 35, 1174-1181.

Zaslavsky, C., Gus, 2002. Idoso: doença cardíaca e comorbidades. Arq. Bras. Cardiol. 79, 635-639. 